\title{
Predicting Isotopomer Distribution in Products of Thermal Cracking with a Kinetic Monte-Carlo Model
}

\author{
HAO XIE ${ }^{1 *}$, MiKe FORMOLO ${ }^{2}$, JOHN EILER ${ }^{1}$
}

${ }^{1}$ Division of Geological and Planetary Sciences, California Institute of Technology, Pasadena, CA, USA (*email address: hxie@caltech.edu)

${ }^{2}$ ExxonMobil Upstream Integrated Solutions Spring, TX, USA

Recently developed analytical methods enable analysis of intramolecular stable isotope distributions of organic compounds in oil and gas, which can serve as signatures of conditions and mechanisms of their formation and destruction. Previously published models of thermal 'cracking' are incapable of predicting the wide range of intramolecular isotope patterns of products because they haven't integrated realistic precursors, elementary reactions and patterns of inheritance. These deficits stem from the complexities of relevant reaction pathways and obscurity of kerogen/bitumen structures.

In this study, we develop a kinetic Monte-Carlo model (kMC) to address this problem. We simulate thermal breakdown of different types of organic matter, including several kerogens, representative oil compounds and oil mixtures. At the onset of each simulation, we initialize the model parent organic molecules with isotopic substitutions, and then subject them to 'cracking' reactions in a many-step process. For each potential elementary step, we determine the rate constants of non-subsitituted positions using an external database that is calibrated to experimental data, and of substituted positions using kinetic isotope effects (KIE) retrieved from either existing first-principle calculations or empirical models calibrated to those calculations. Every simulation captures a possible route of thermal degradation and tallies the numbers of each unique isotopomer at the end. We reach statistically meaningful results of isotopomer ratios by repeating a sufficient number of such simulations.

This model output includes proportions of many of the isotopomers of every C1-C7 hydrocarbon. We show that molecular structure of the parent material exerts an important influence over the intramolecular isotope distribution of its products. For instance, straight chain structures lead to carbon and hydrogen isotope depletion on the terminal positions of $\mathrm{C} 3+$ alkanes. We compare our model results with a global dataset of compound-specific and position-specific isotope measurements of $\mathrm{C} 1-\mathrm{C} 5$ alkanes to provide insights on the formation of gases. 